So ist, hoffe ich, ein Anfängerlehrbuch entstanden, das in vielen Einzelheiten vom Herkömmlichen abweicht und das auf verhăltnismäßig begrenztem Raum von 618 Seiten auf moderner Grundlage einen umfangreichen Wissensstoff in weitgehend vollständiger und didaktisch abgewogener Darstellung vermittelt. Im Zusammenwirken mit dem Verlag, dem ich für sein Eingehen auf alle meine Wünsche bestens zu danken habe wurde es dabei ermöglicht, das Buch trotz der völligen Umgestaltung des Stoffs, der Neuausstattung mit Abbildungen, des vergrößerten Seitenformats, der Vermehrung um 123 Textseiten und der gediegenen Druck- und Papierausstattung zum bisherigen, an sich schon niedrigen Preis herauszubringen.

Herzlichen Dank schulde ich den Münchener Kollegen Prof. Dr. O. Hönıcscimid, Prof. Dr. F. Klages, Dozent Dr. H. Lux und Dr.-Ing. O. Stecher für zahlreiche Anregungen und Hinweise beim Lesen der Korrekturen. Ebenso danke ich meiner lieben Frau für ihre unermüdliche Mitarbeit bei der Anfertigung des umfangreichen Registers.

München, im November 1942.

\title{
Vorwort zur 24. und 25. Auflage
}

Die inı Juni 1943 herausgekommene, völlig neubearbeitete 22.-23. Auflage des vorliegenden Lehrbuches war bereits nach wenigen Tagen vergriffen, so daß sofort mit der Bearbeitung zweier weiterer Auflagen begonnen werden mußte. Diese beiden Auflagen verbrannten kurz nach ihrer Fertigstellung Ende 1943 bei einem Terrorangriff. Dabei ging leider auch der ganze Satz samt allen Klischees für die Abbildungen verloren. Dank der Tatkraft des Verlags gelang es aber, das Werk mit allen seinen Abbildungen in überraschend kurzer Zeit wieder neu zu setzen und auch dieses Mal in vorbildlicher Druck- und Papierausstattung herauszubringen.

In Anbetracht der Kürze der für die eigentliche U'berarbeitung zur Verfügung ste. henden Zeit wurde von größeren Änderungen abgesehen, zumal aus zahlreichen den Verlag und dem Verfasser zugegangenen Zuschiriften herrorging, da $ß$ das Buch in seiner neuen Form allgemeine Zustimmung gefunden hat. Dementsprechend habe ich mich danit begnügt, die neue Auflage einer sorgfältigen Durchsicht zu unterziehen, Unklarheiten im Text zu beseitigen, notwendige Ergänzungen einzufügen, Abbildungen zu verbessern, die Seitenverweisungen im Text zu vermehren, die Nomenklatur zu bereinigen und das Register stark zu erweitern. Der Umfang des Buches verringerte sich infolge Wahl einer anderen Drucktype trotz vermehrten Inhalts um 74 Textseiten.

Für Anregungen und Zuschriften danke ich den Kollegen Ebert.Wien, FreseniusWiesbaden, Freudenberg - Heidelberg, Frick e - Stuttgart, Goubenu - Göttingen, Hecht Graz, Hieber - München, Hofmanw - Wien, Hürtig-Prag, JAHR-Berlin, JuzA - Heidel. berg, Klement - Prag, Klemm - Danzig, Kuss-Duisburg, Nestre - Karlsruhe, PhilippiInnsbruck, Pohland-Berlin, Rienäcker-Rostock, SchenK-Königsberg, Schmeisser- 
Königsberg, Scholder-Karlsruhe, Sschwarz-Königsberg, Sрӥтн-Wien, Stock - Berlin, Thieler-Frankfurt a. M., Wurstzr-Ludwigshafen. Ihre Anregungen werden, soweit sie nicht schon in der vorliegenden Neuauflage Platz gefunden haben, bei einer kommenden Auflage berücksichtigt werden.

Die im Text mit II bezeichneten Seitenverweise beziehen sich auf die 25. Auflage des zweiten (organischen) Teils des Lehrbuchs der Chemie von A. F. Holleman. Die Seitenzahlen des umfangreichen Registers wurden, um das Erscheinen der neuen Auflage nicht noch weiter hinauszuzögern, der Einfachheit halber nach einem durch die verminderte Seitenzahl gegebenen Ubertragungsschlüssel abgeändert. Sie sind daher gelegentlich um 1 zu erhöhen oder zu erniedrigen.

Münohen, im November 1944.

Egon Wiberg. 